\title{
Amino Acid Based Cationic Surfactants in Aqueous Solution: Physicochemical Study and Application of Supramolecular Chirality in Ketone Reduction
}

\author{
Sangita Roy, Debapratim Das, Antara Dasgupta, Rajendra Narayan Mitra, \\ and Prasanta Kumar Das* \\ Department of Biological Chemistry, Indian Association for the Cultivation of Science, \\ Jadavpur, Kolkata 700032, India
}

\section{Supporting information}

\section{Theoretical Formulation for the Minimum Energy Configuration.}

a) in the plane of $a$ and $b$ groups:

$$
\begin{gathered}
{\left[\mathrm{u} / \mathrm{k}_{\mathrm{B}} \mathrm{T}\right]_{\mathrm{a}, \mathrm{b}}=\left[\mathrm{u}_{\mathrm{a}, \mathrm{a}} / \mathrm{k}_{\mathrm{B}} \mathrm{T}\right]_{\mathrm{a}, \mathrm{a}}+\left[\mathrm{u}_{\mathrm{b}, \mathrm{b}} / \mathrm{k}_{\mathrm{B}} \mathrm{T}\right]_{\mathrm{b}, \mathrm{b}}} \\
=(4 / \mathrm{T})\left(\varepsilon_{\mathrm{a}} / \mathrm{k}_{\mathrm{B}}\right)\left[\left(\mathrm{r} / \sigma_{\mathrm{a}}+1 / 2 \cos \left(\Phi_{2}-\alpha\right)-1 / 2 \sin \left(\Phi_{2}-\alpha\right) \cot \left(\Phi_{1}-\alpha\right)\right)^{-12}-\left(\mathrm{r} / \sigma_{\mathrm{a}}+1 / 2 \cos \left(\Phi_{2}-\alpha\right)-\right.\right. \\
\left.\left.1 / 2 \sin \left(\Phi_{2}-\alpha\right) \cot \left(\Phi_{1}-\alpha\right)\right)^{-6}\right]+(4 / \mathrm{T})\left(\varepsilon_{\mathrm{b}} / \mathrm{k}_{\mathrm{B}}\right)\left[\left(\mathrm{r} / \sigma_{\mathrm{b}}-1 / 2 \cos \left(\beta-\Phi_{1}-\alpha\right)-1 / 2 \sin \left(\beta-\Phi_{1}-\alpha\right) \cot (\beta-\right.\right. \\
\left.\left.\left.\Phi_{2}\right)\right)^{-12}-\left(\mathrm{r} / \sigma_{\mathrm{b}}-1 / 2 \cos \left(\beta-\Phi_{1}-\alpha\right)-1 / 2 \sin \left(\beta-\Phi_{1}-\alpha\right) \cot \left(\beta-\Phi_{2}\right)\right)^{-6}\right]
\end{gathered}
$$

Here, $\sigma_{\mathrm{a}}$ and $\sigma_{\mathrm{b}}$ are the effective diameters of the respective groups. $\alpha$ is the orientation of the line joining the two adjacent chiral centers, and $\mathrm{r}$ is the distance between them. $\Phi_{1}$ and $\Phi_{2}$ are the orientations of neighbouring $\boldsymbol{a}$ or $\boldsymbol{b}$ groups. $\beta$ is the angle between $\boldsymbol{a}$ and $\boldsymbol{b}$ groups(see Figure 2 in reference $11 \mathrm{a}$ in text). $\varepsilon_{\mathrm{a}}$ and $\varepsilon_{\mathrm{b}}$ are the Lennard-Jones energy parameters for $\boldsymbol{a}$ and $\boldsymbol{b}$. $\mathrm{k}_{\mathrm{B}}$ is the Boltzman constant. A linear dependence was found between this $\varepsilon_{\mathrm{LJ}}$ and size of the groups from Ben-Amotz and Herschbach calculation. For $1 \AA \AA$ increment in the effective diameter of each group, the $\varepsilon_{\mathrm{LJ}} / \mathrm{k}_{\mathrm{B}}$ value is assumed to increase $\sim 100 \mathrm{~K}$

$\sigma_{\mathrm{a}}$ and $\sigma_{\mathrm{b}}$ can be calculated from the following equation: 


$$
\begin{gathered}
\mathrm{V}_{\mathrm{hs}}=1.086\left(\mathrm{~V}_{\mathrm{s}}-9.94\right) \\
\sigma=1.244\left(\mathrm{~V}_{\mathrm{hs}}\right)^{1 / 3}
\end{gathered}
$$

where $V_{s}$ is the "space-filling" volume, whichis calculated from the group increments listed by Bondi. 


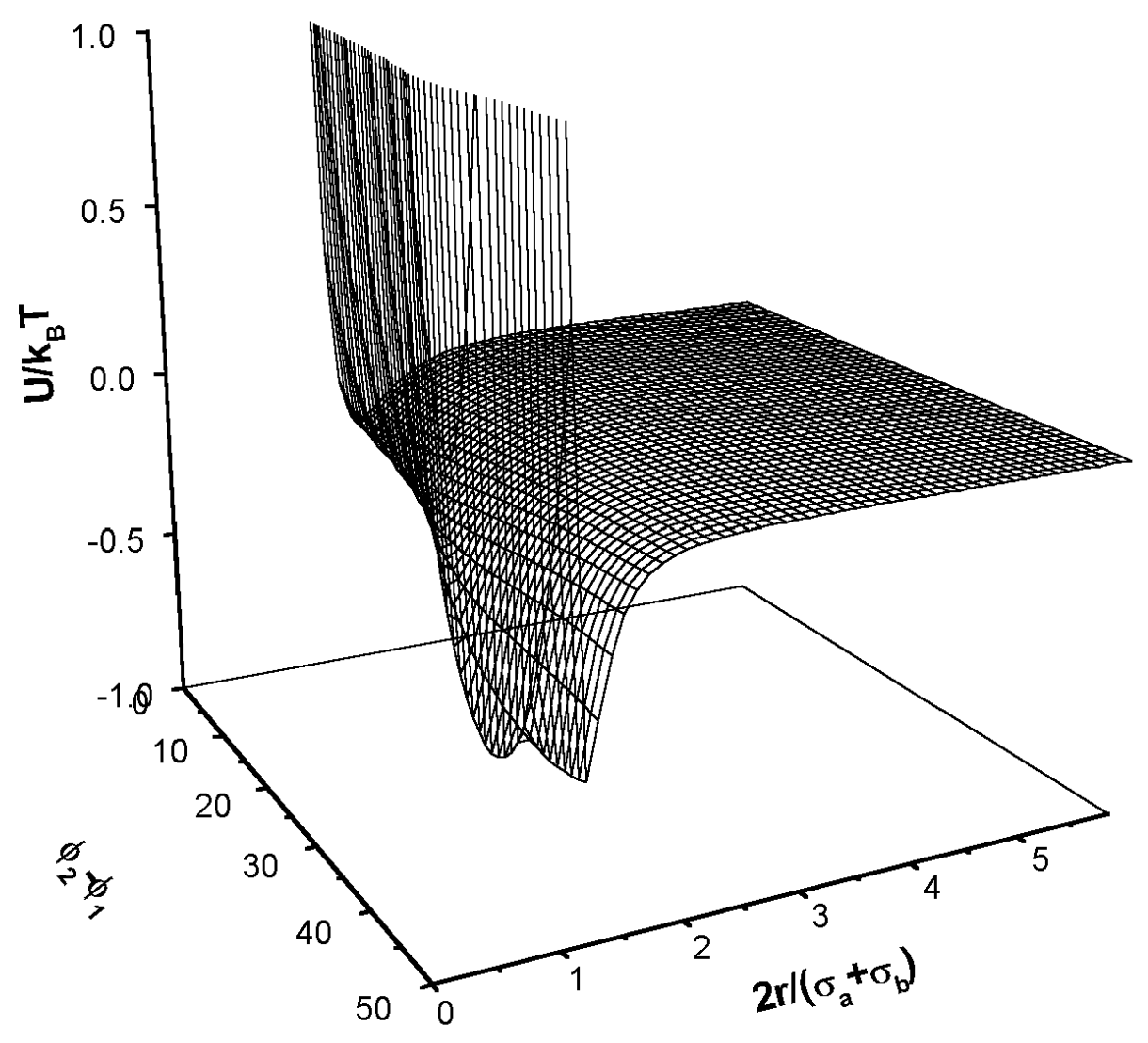

Effective Pair Potential for a pair molecule of surfactant $\mathbf{1}$ $\sigma_{\mathrm{a}}=1.4 \AA \dot{A}, \sigma_{\mathrm{b}}=2.99 \AA ⿱ ㇒ 㠯, \varepsilon_{\mathrm{a}} / \mathrm{k}_{\mathrm{B}}=140 \mathrm{~K}, \varepsilon_{\mathrm{b}} / \mathrm{k}_{\mathrm{B}}=299 \mathrm{~K}, \beta=110^{\circ}, \alpha=0^{0}$ 


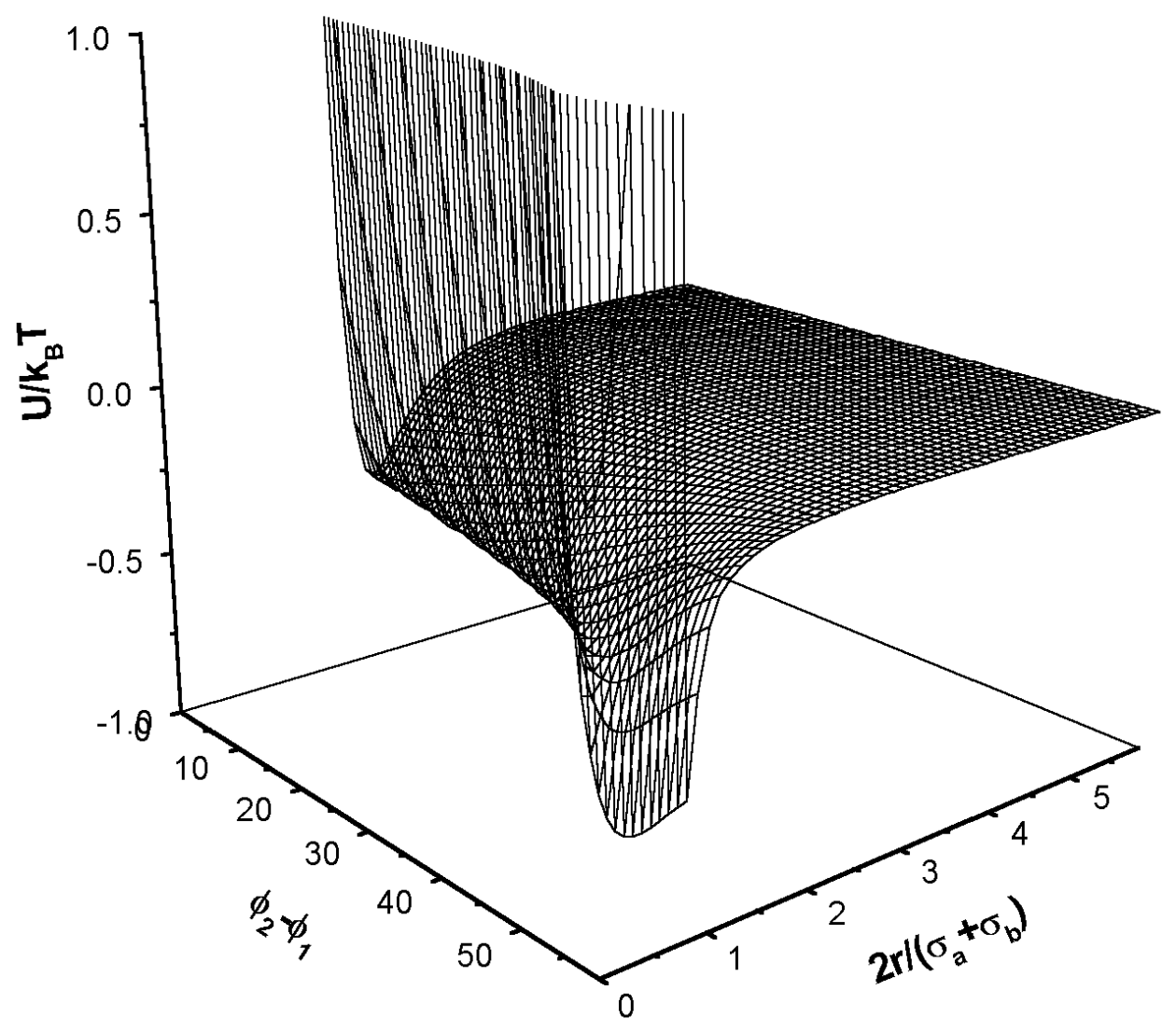

Effective Pair Potential for a pair molecule of surfactant 2

$\sigma_{\mathrm{a}}=1.4 \AA \hat{,} \sigma_{\mathrm{b}}=5.58 \AA, \varepsilon_{\mathrm{a}} / \mathrm{k}_{\mathrm{B}}=140 \mathrm{~K}, \varepsilon_{\mathrm{b}} / \mathrm{k}_{\mathrm{B}}=558 \mathrm{~K}, \beta=110^{\circ}, \alpha=0^{0}$ 


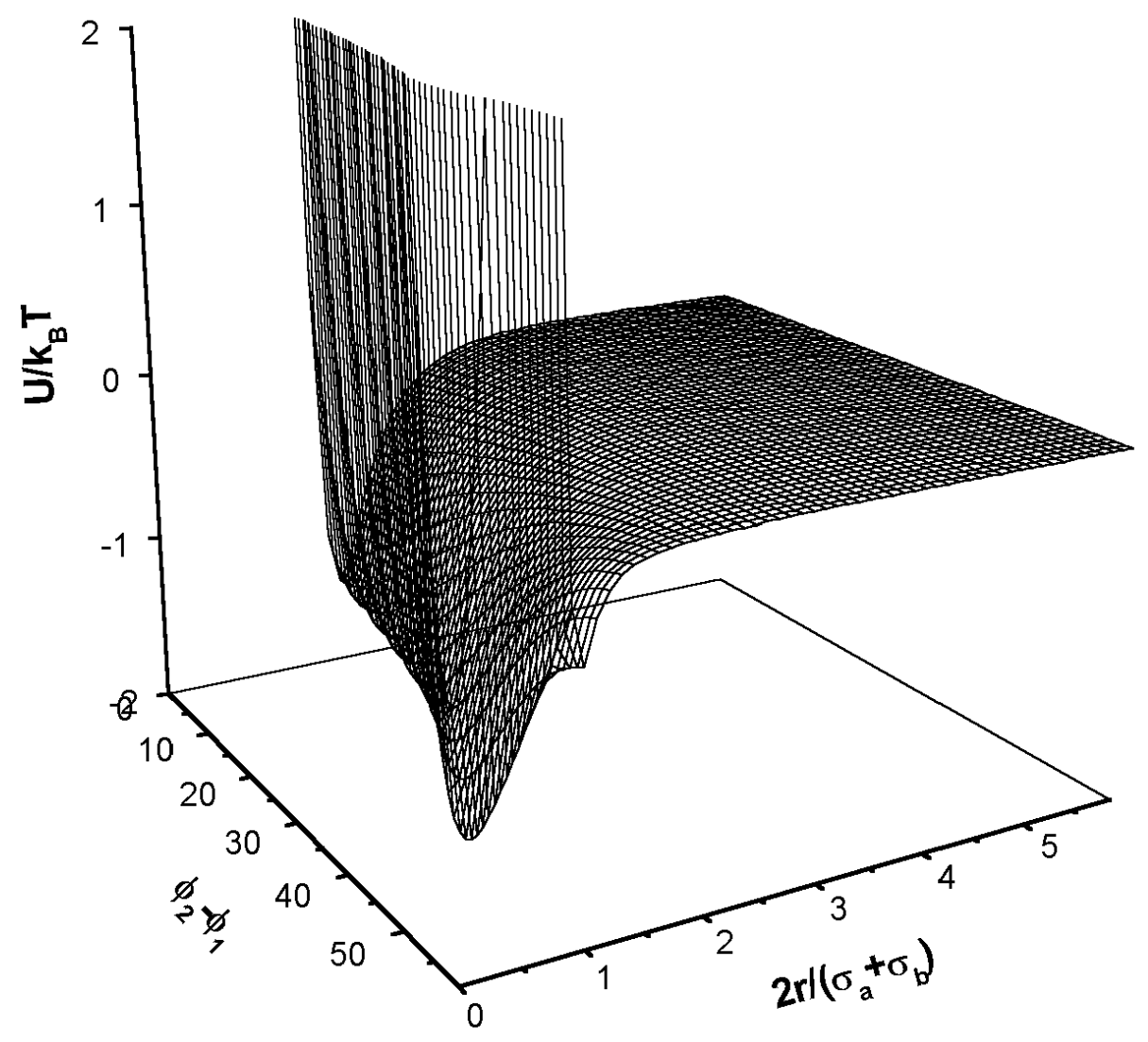

Effective Pair Potential for a pair molecule of surfactant 3

$\sigma_{\mathrm{a}}=1.4 \AA, \sigma_{\mathrm{b}}=4.4 \AA, \varepsilon_{\mathrm{a}} / \mathrm{k}_{\mathrm{B}}=140 \mathrm{~K}, \varepsilon_{\mathrm{b}} / \mathrm{k}_{\mathrm{B}}=440 \mathrm{~K}, \beta=110^{\circ}, \alpha=0^{0}$ 
HPLC chromatograms for micelle mediated asymmetric reduction of prochiral ketones (mobile phase: hexane/isopropanol 95/5, v/v; $\lambda=254 \mathrm{~nm}$; flow rate: $0.5 \mathrm{~mL} / \mathrm{min}$ ) :
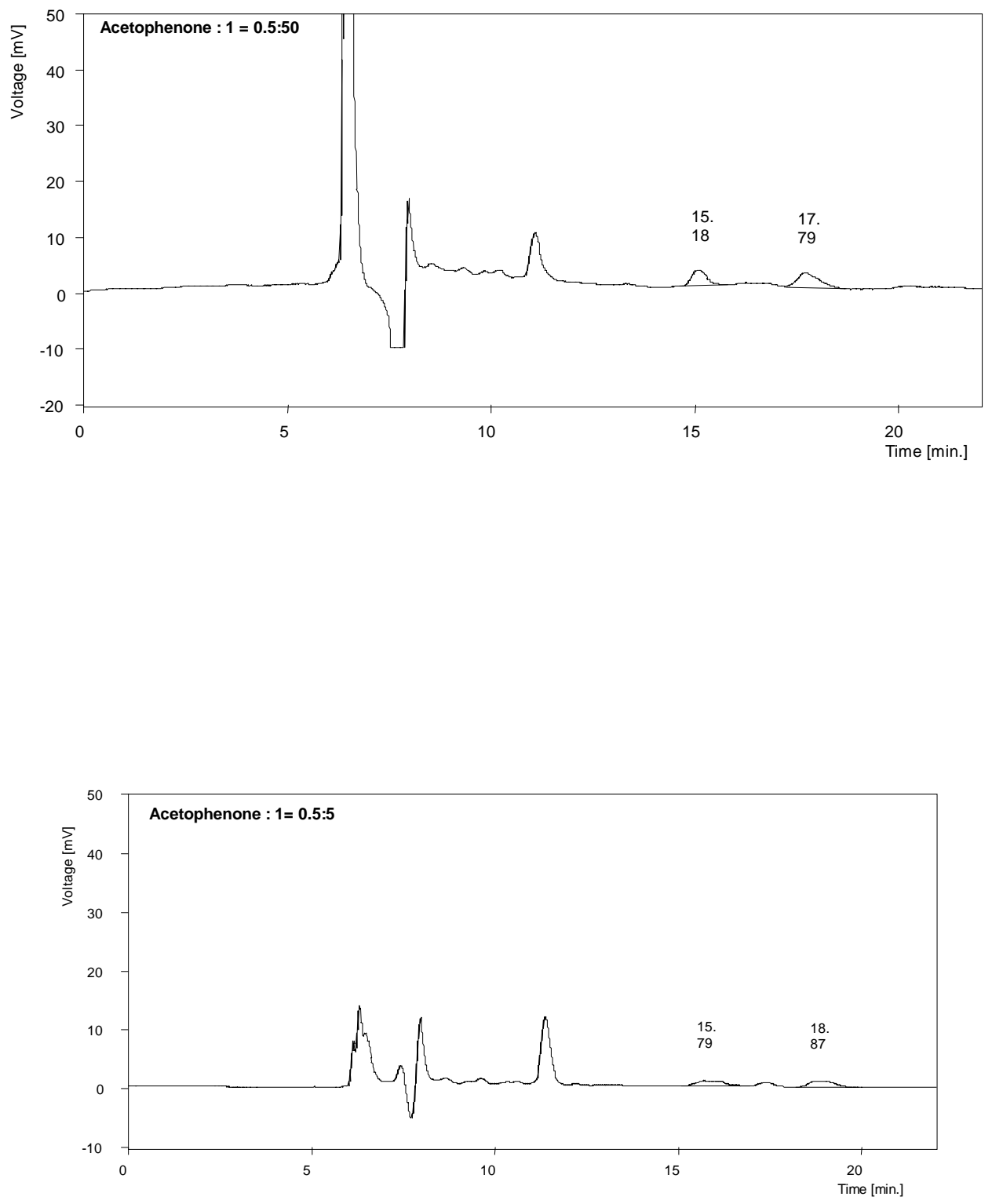

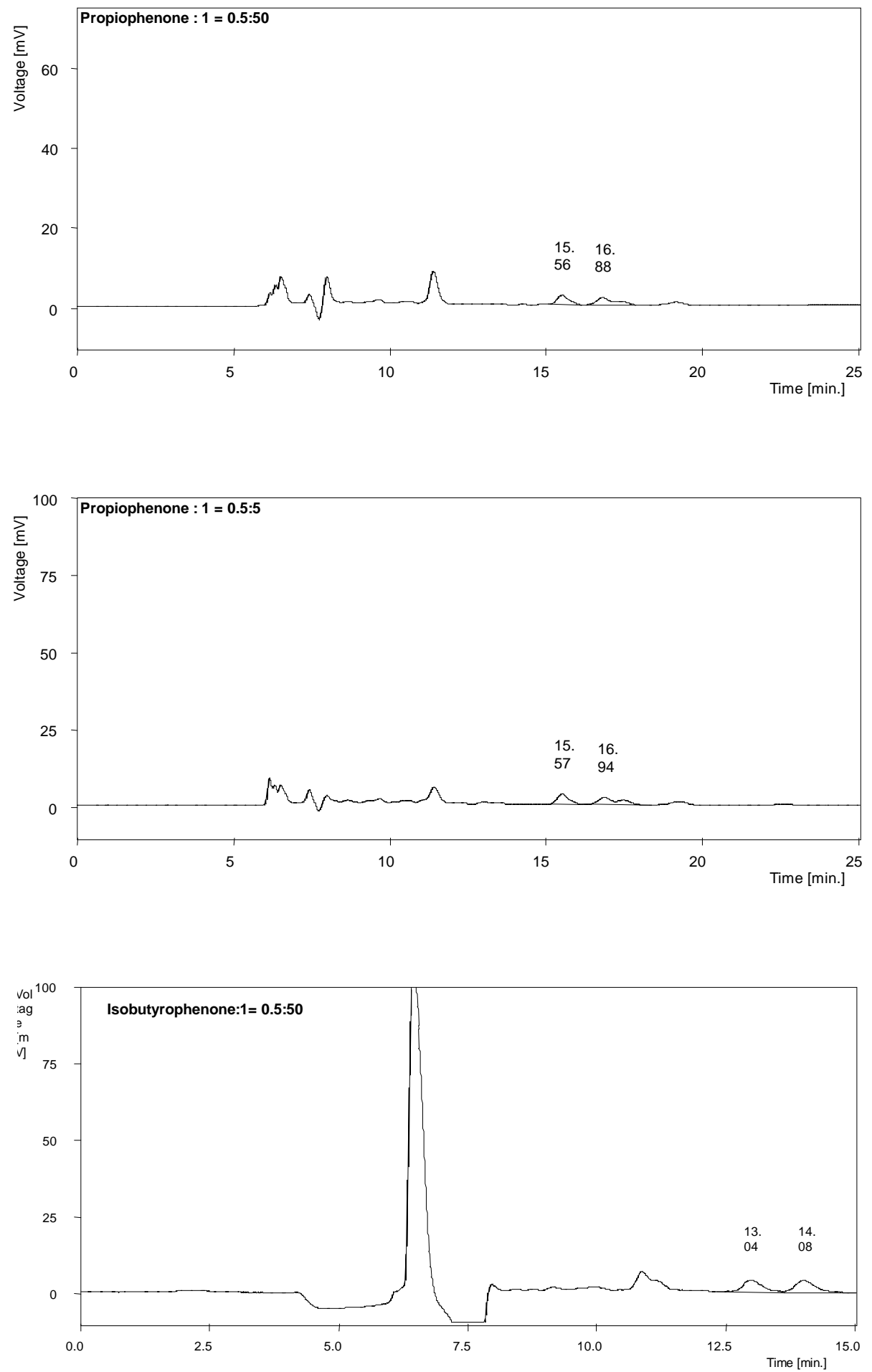

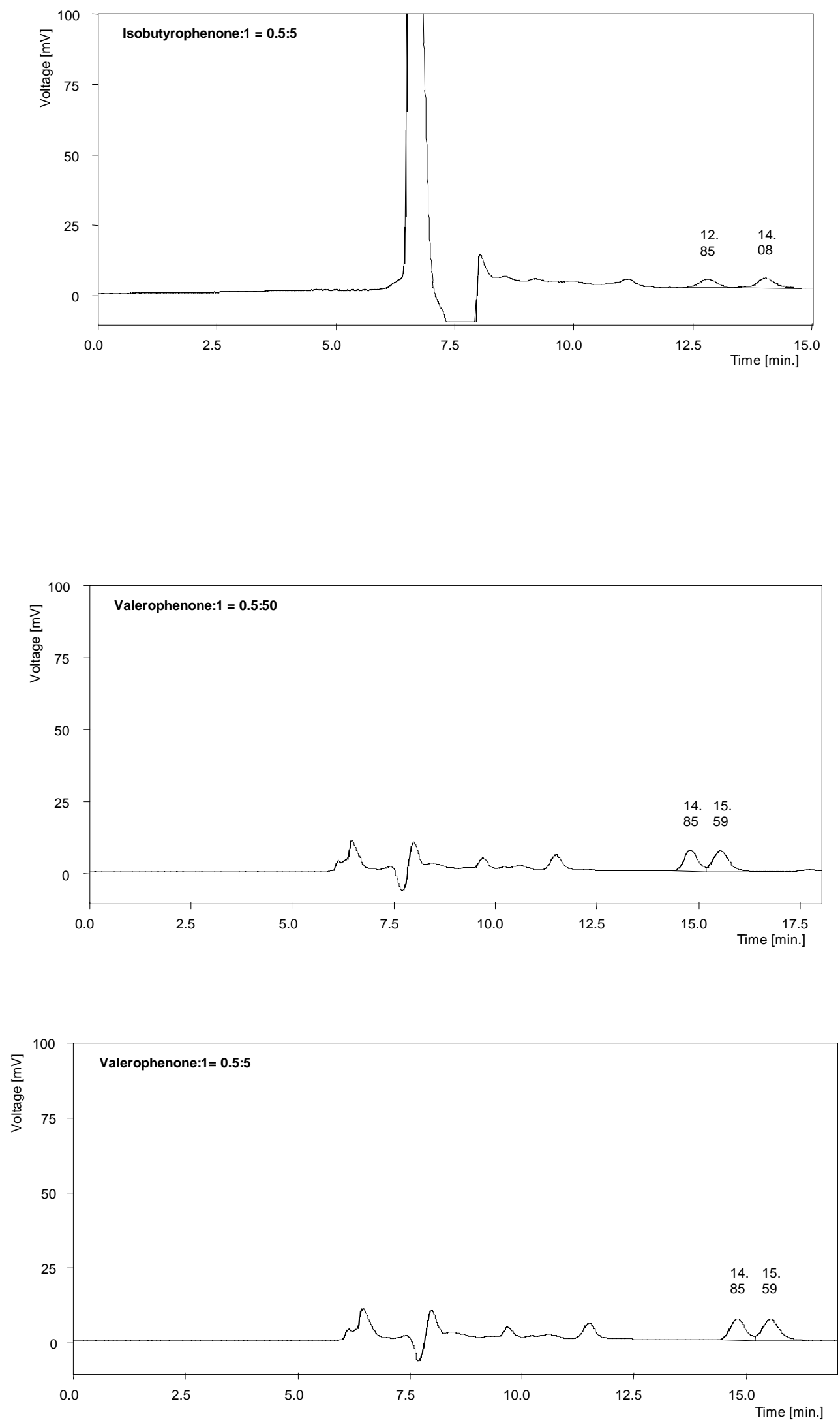

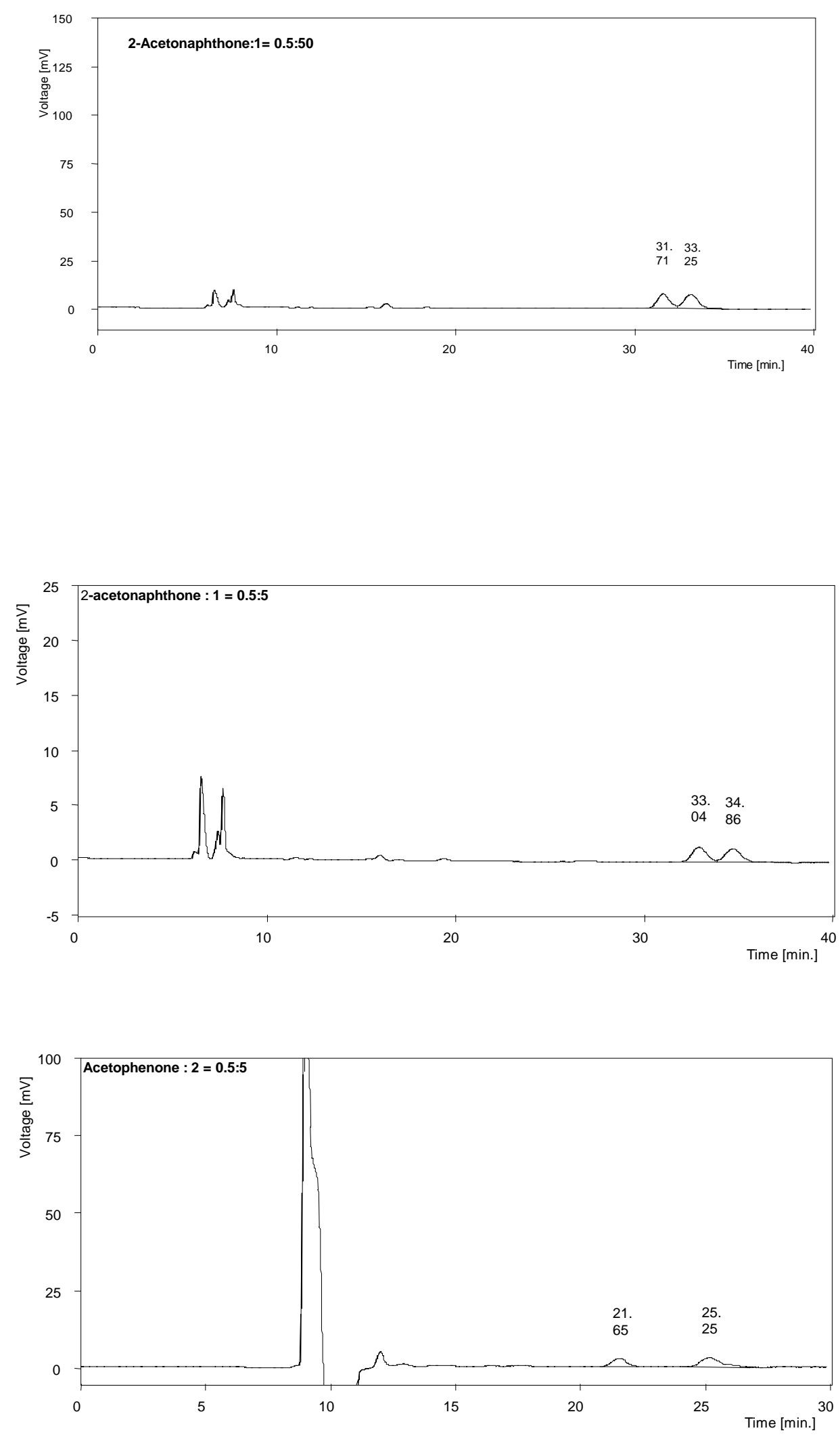

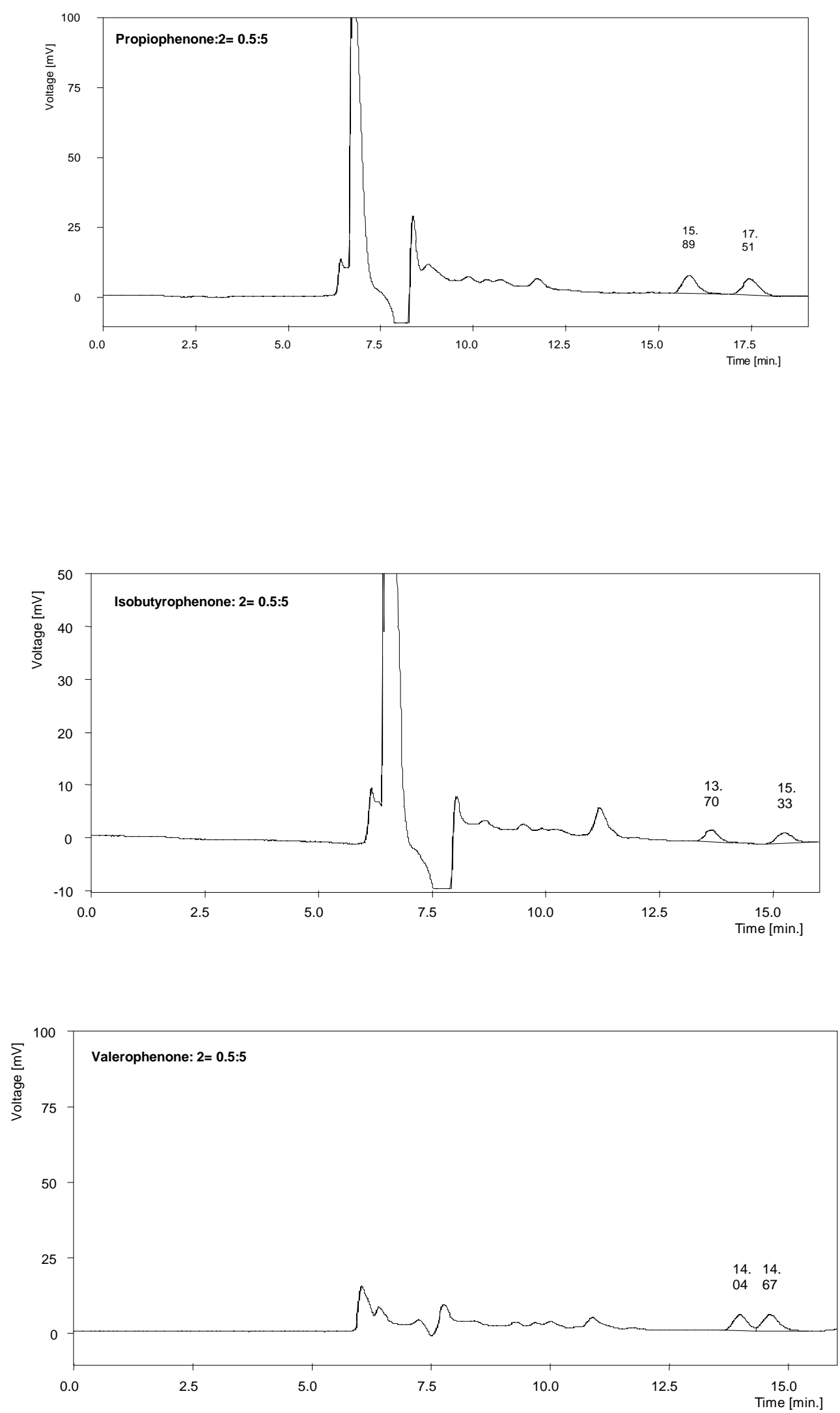

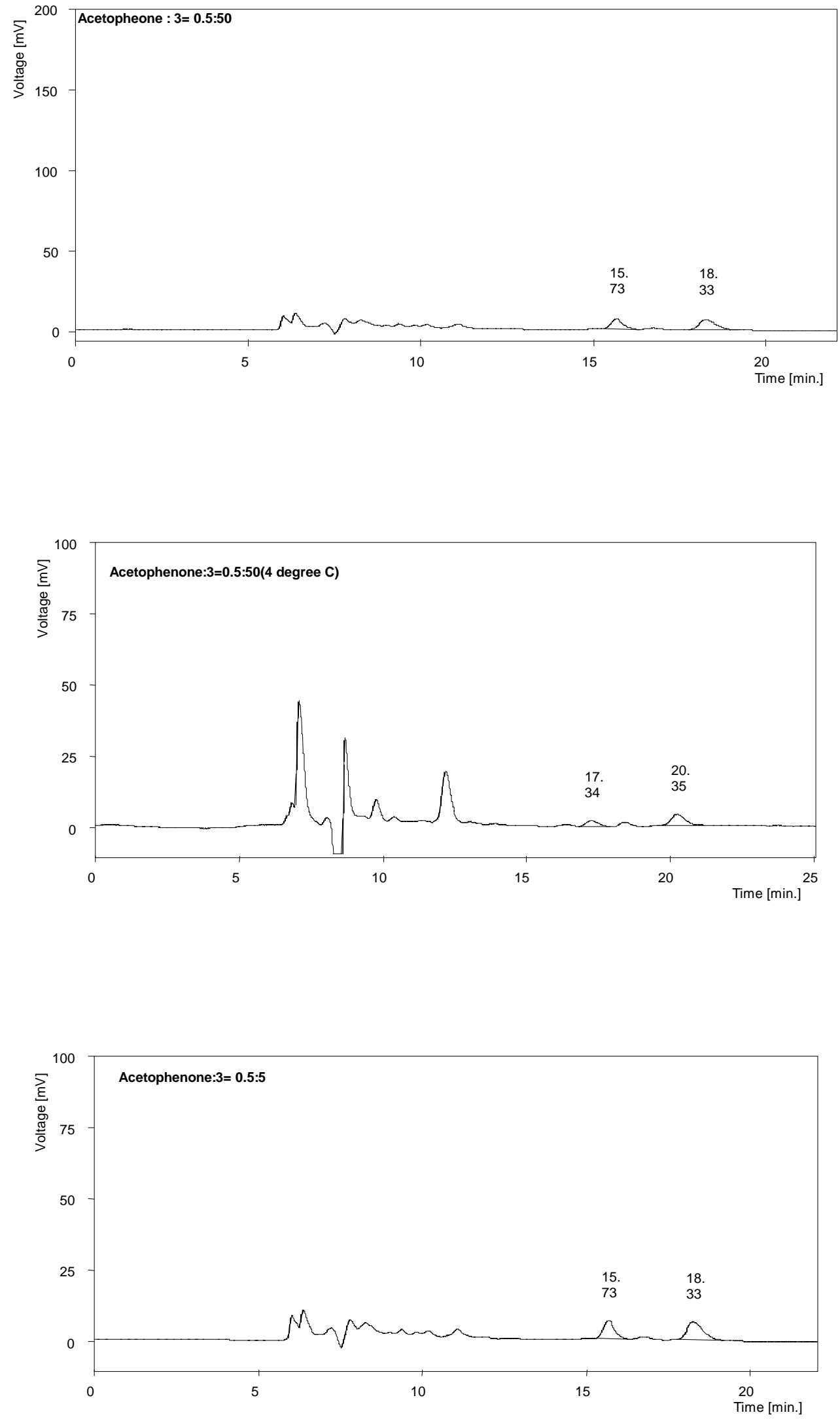

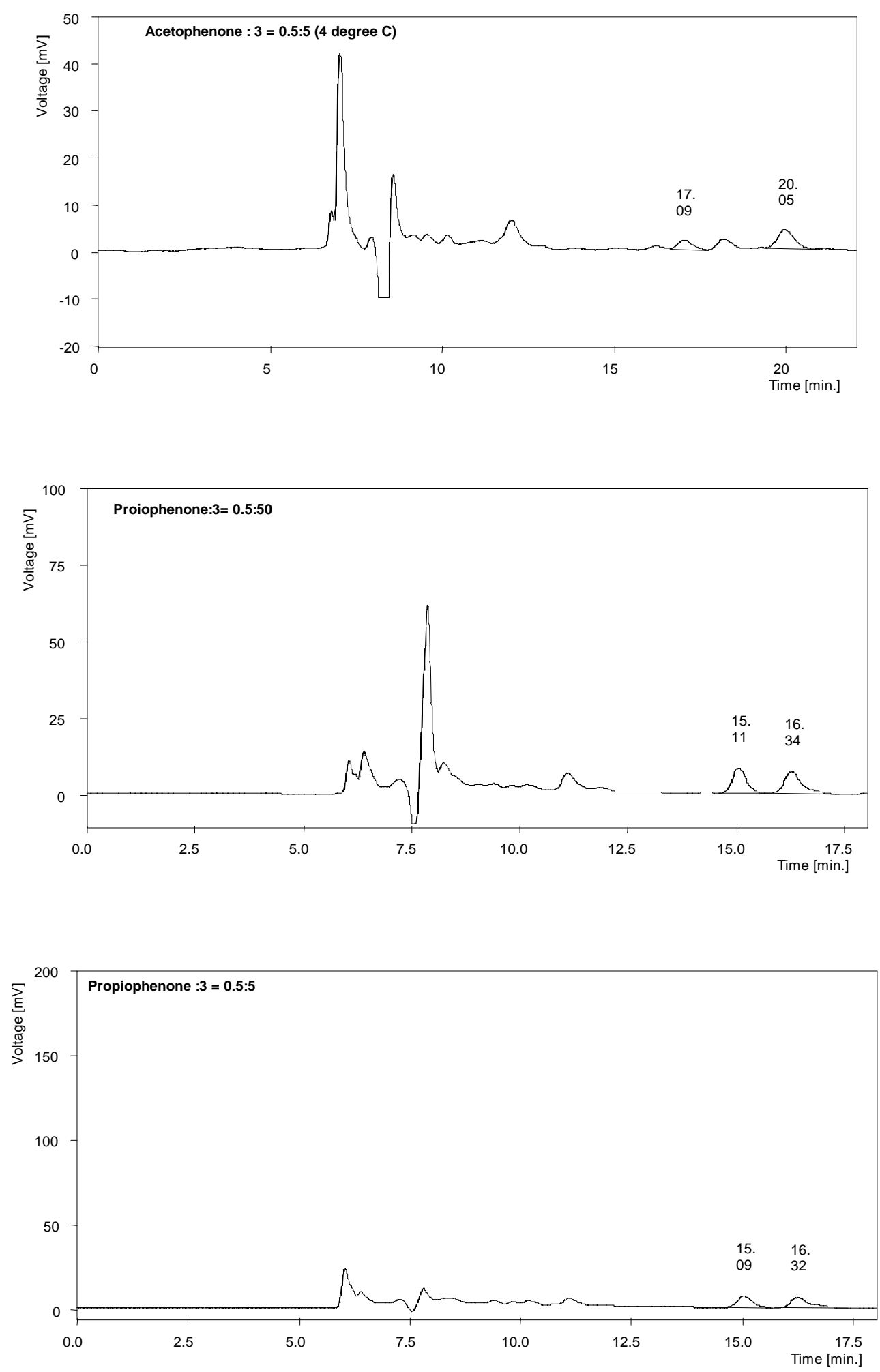

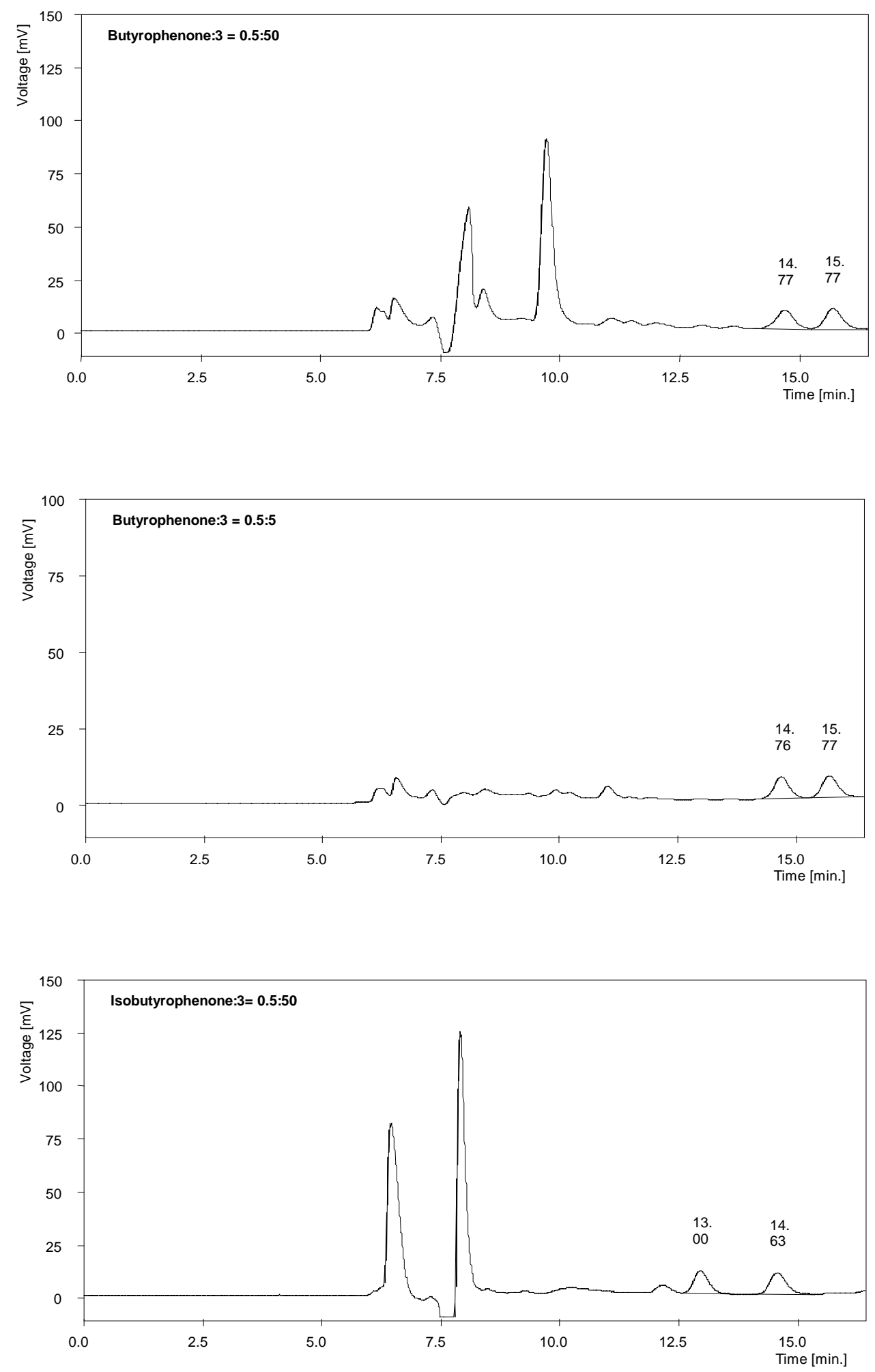

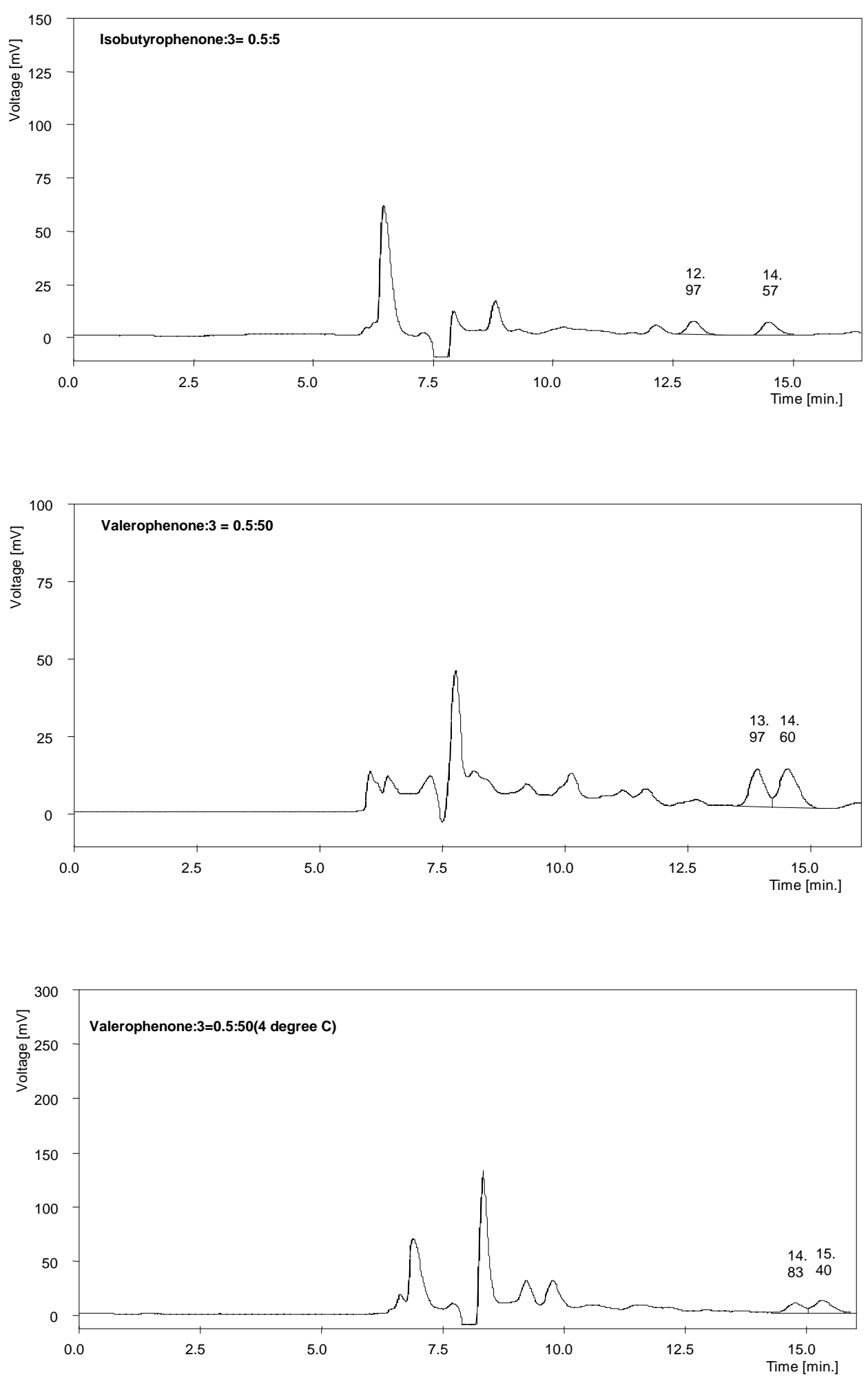

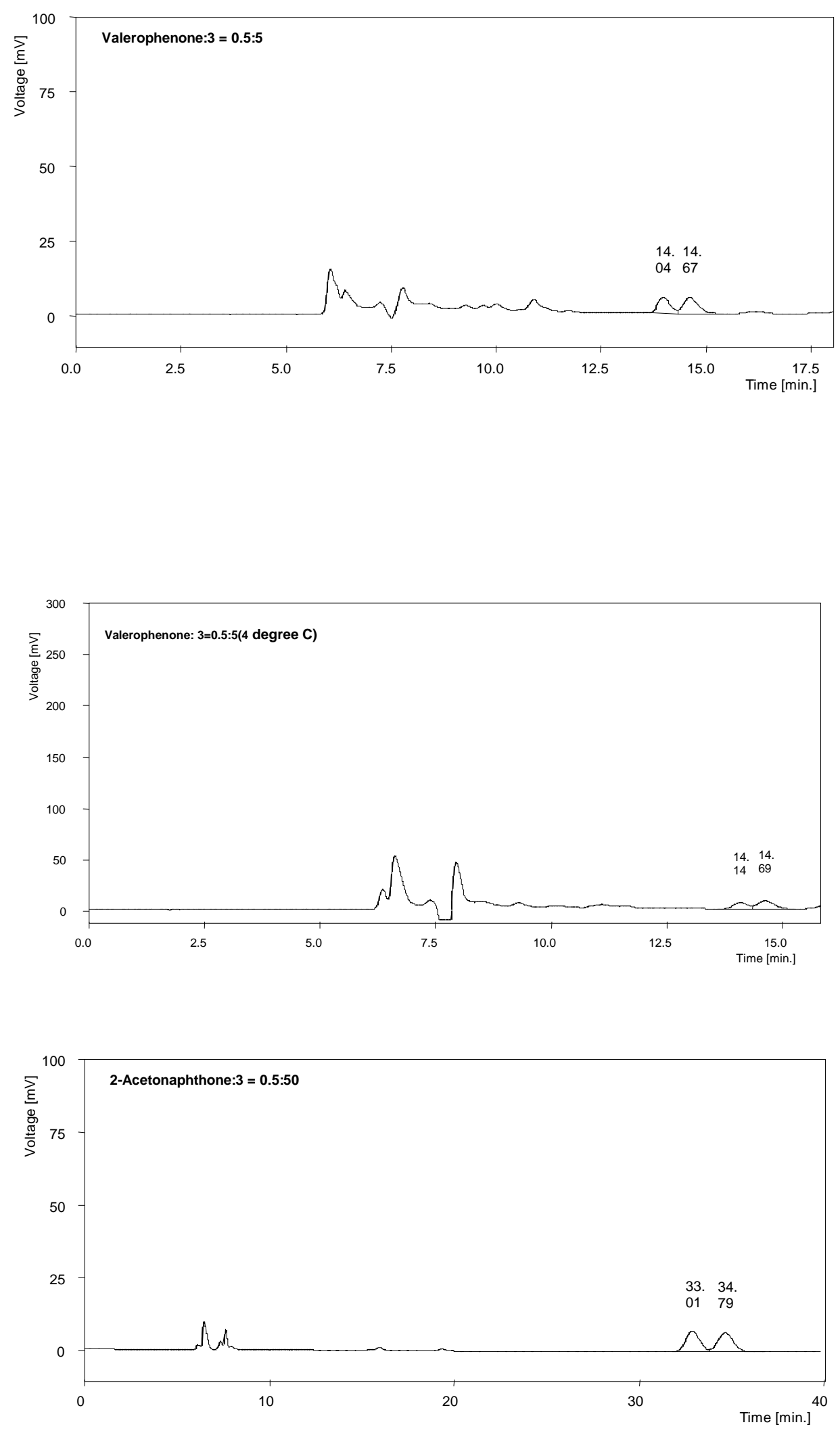

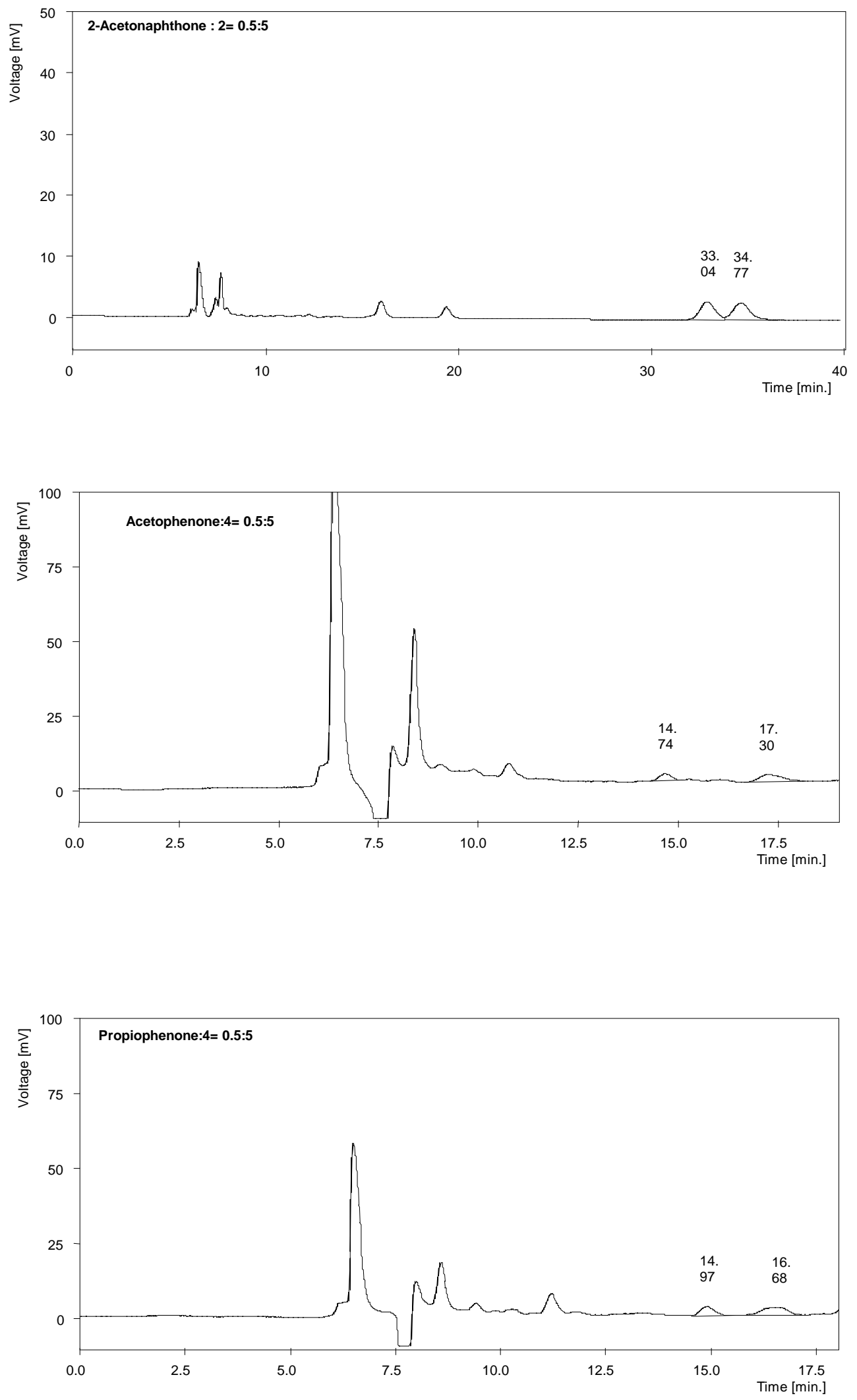

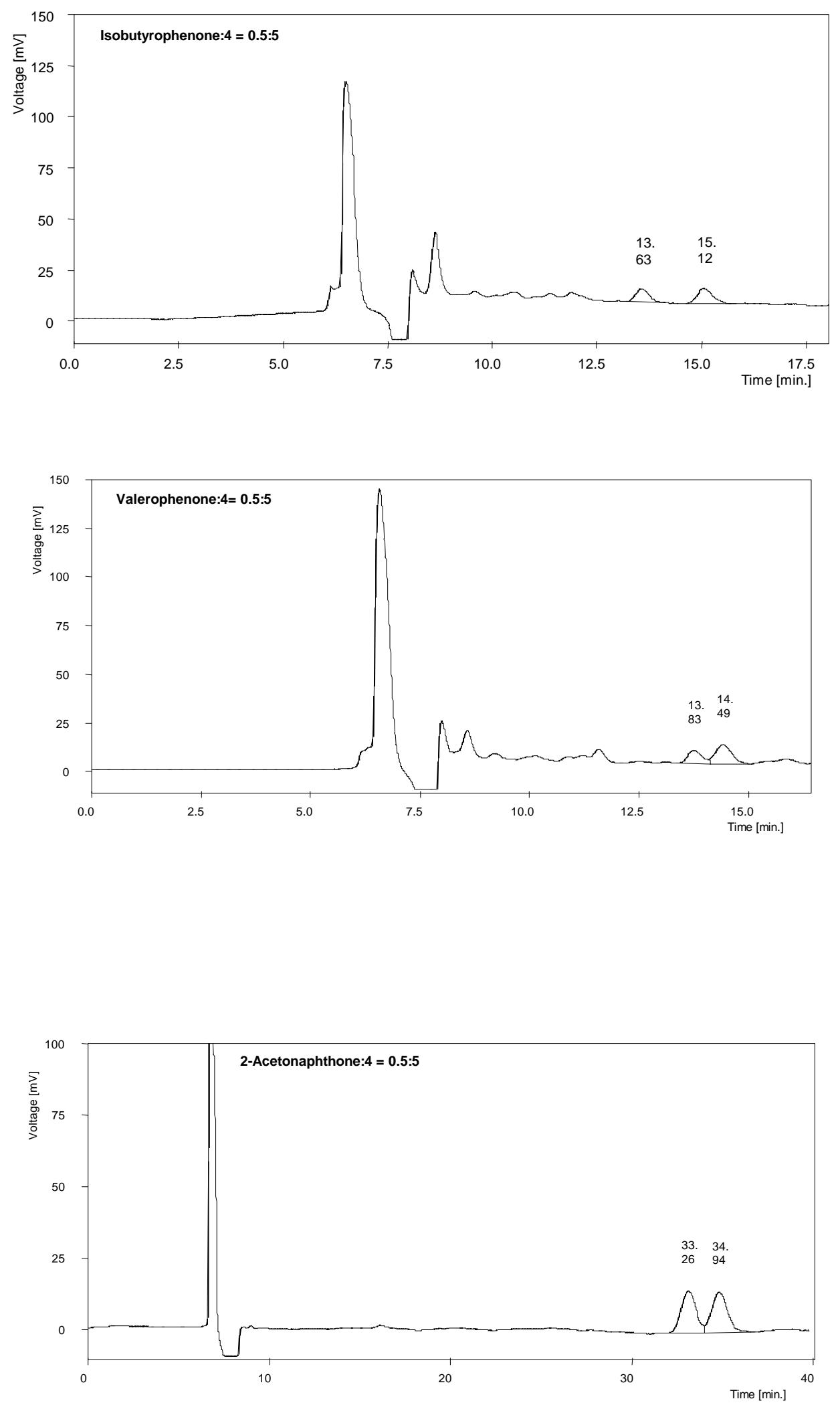

Table S1. HPLC calibration equation of the pure alcohols, peak area, and yield of micelle mediated reduction of prochiral ketones ${ }^{\mathrm{a}, \mathrm{b}}$

\begin{tabular}{|c|c|c|c|c|c|c|c|c|c|c|c|c|c|c|c|}
\hline \multirow[b]{3}{*}{ Ketone } & \multirow{3}{*}{$\begin{array}{l}\text { Calibration } \\
\text { Equation of } \\
\text { alcohols for } \\
\text { corresponding } \\
\text { ketones } \\
\text { Area }(\mu \mathrm{V})= \\
\end{array}$} & \multirow{3}{*}{$\begin{array}{l}\text { Corr. } \\
\text { Coeff. }\end{array}$} & \multirow[b]{3}{*}{$\begin{array}{l}{[\text { Surfactant }]} \\
\quad(\mathrm{mM})\end{array}$} & \multirow{2}{*}{\multicolumn{2}{|c|}{$\begin{array}{c}\text { I } \\
\text { Peak Area }(\mu V)\end{array}$}} & \multirow[b]{3}{*}{$\begin{array}{l}\text { Yield } \\
(\%)\end{array}$} & \multirow{2}{*}{\multicolumn{2}{|c|}{$\begin{array}{c}\text { II } \\
\text { Peak Area }(\mu V) \\
\end{array}$}} & \multirow[b]{3}{*}{$\begin{array}{l}\text { Yield } \\
(\%)\end{array}$} & \multirow{2}{*}{\multicolumn{2}{|c|}{$\begin{array}{r}\text { III } \\
\text { Peak Area }(\mu \mathrm{V}) \\
\end{array}$}} & \multirow[b]{3}{*}{$\begin{array}{l}\text { Yield } \\
(\%)\end{array}$} & \multirow{2}{*}{\multicolumn{2}{|c|}{$\begin{array}{c}\text { IV } \\
\text { Peak Area }(\mu \mathrm{V})\end{array}$}} & \multirow[b]{3}{*}{$\begin{array}{l}\text { Yield } \\
(\%)\end{array}$} \\
\hline & & & & & & & & & & & & & & & \\
\hline & & & & $\mathrm{R}$ & S & & $\mathrm{R}$ & $\mathrm{S}$ & & $\mathrm{R}$ & $\mathrm{S}$ & & $\mathrm{R}$ & $\mathrm{S}$ & \\
\hline \multirow[t]{2}{*}{ Acetophenone } & \multirow[t]{2}{*}{$\begin{array}{c}4 \times 10^{8}[\text { alcohol }] \\
\mathrm{M}+2872.3\end{array}$} & \multirow[t]{2}{*}{0.999} & \multirow[t]{2}{*}{50} & 155500 & 167784 & 64 & 156751 & 203596 & 72 & $\begin{array}{l}165577 \\
75850^{c}\end{array}$ & $\begin{array}{l}210734 \\
140864^{\mathrm{c}}\end{array}$ & $\begin{array}{l}75 \\
44^{c}\end{array}$ & \multirow[t]{2}{*}{150250} & \multirow[t]{2}{*}{272989} & \multirow[t]{2}{*}{84} \\
\hline & & & & 150400 & 156539 & 61 & & & & $\begin{array}{l}153219 \\
65299^{c}\end{array}$ & $\begin{array}{l}212454 \\
157120^{\mathrm{c}}\end{array}$ & $\begin{array}{l}73 \\
42\end{array}$ & & & \\
\hline Propiophenone & $\begin{array}{c}3 \times 10^{8}[\text { alcohol }] \\
\mathrm{M}+4752\end{array}$ & 0.997 & $\begin{array}{c}50 \\
5\end{array}$ & $\begin{array}{l}100794 \\
83922\end{array}$ & $\begin{array}{c}108757 \\
96168\end{array}$ & $\begin{array}{l}55 \\
48\end{array}$ & 160923 & 169854 & 87 & $\begin{array}{l}149532 \\
132891\end{array}$ & $\begin{array}{l}168960 \\
155714\end{array}$ & $\begin{array}{l}83 \\
76\end{array}$ & 120850 & 195511 & 83 \\
\hline Butyrophenone & $\begin{array}{c}3 \times 10^{8} \text { [alcohol] } \\
\mathrm{M}+16949\end{array}$ & 0.986 & $\begin{array}{c}50 \\
5\end{array}$ & $\begin{array}{l}136556 \\
140274\end{array}$ & $\begin{array}{l}138758 \\
141684\end{array}$ & $\begin{array}{l}69 \\
71\end{array}$ & 132751 & 134622 & 67 & $\begin{array}{l}135615 \\
139425\end{array}$ & $\begin{array}{l}138909 \\
142526\end{array}$ & $\begin{array}{l}69 \\
71\end{array}$ & 135468 & 136829 & 68 \\
\hline Isobutyrophenone & $\begin{array}{c}3 \times 10^{8} \\
\text { [alcohol] } \mathrm{M}+ \\
3948.7\end{array}$ & 0.9989 & $\begin{array}{c}50 \\
5\end{array}$ & $\begin{array}{l}141405 \\
129560\end{array}$ & $\begin{array}{l}150754 \\
160933\end{array}$ & $\begin{array}{l}77 \\
76\end{array}$ & 131635 & 144040 & 72 & $\begin{array}{l}141847 \\
127818\end{array}$ & $\begin{array}{l}152136 \\
146427\end{array}$ & $\begin{array}{l}77 \\
69\end{array}$ & 92114 & 107634 & 54 \\
\hline Valerophenone & $\begin{array}{c}4 \times 10^{8}[\text { alcohol] } \\
\mathrm{M}+3261.7\end{array}$ & 0.999 & 50 & 172366 & 194434 & 73 & 173666 & 184408 & 71 & $\begin{array}{l}156170 \\
85111^{\mathrm{c}}\end{array}$ & $\begin{array}{l}204500 \\
130359^{\mathrm{c}}\end{array}$ & $\begin{array}{l}71 \\
42^{\mathrm{c}}\end{array}$ & 164837 & 259114 & 84 \\
\hline & & & 5 & 160470 & 186334 & 70 & & & & $\begin{array}{l}111228 \\
66221^{\mathrm{c}}\end{array}$ & $\begin{array}{l}131305 \\
97287^{\circ}\end{array}$ & $\begin{array}{l}48 \\
32^{\mathrm{c}}\end{array}$ & & & \\
\hline 2-Acetonaphthone & $\begin{array}{c}1 \times 10^{10} \\
\text { [alcohol] M- } \\
3450.2\end{array}$ & 0.999 & $\begin{array}{c}50 \\
5\end{array}$ & $\begin{array}{l}390450 \\
378380\end{array}$ & $\begin{array}{l}408833 \\
395403\end{array}$ & $\begin{array}{l}64 \\
62\end{array}$ & 138456 & 389330 & 62 & $\begin{array}{l}382484 \\
380400\end{array}$ & $\begin{array}{c}39200 \\
396720\end{array}$ & $\begin{array}{l}63 \\
62\end{array}$ & 380450 & 472314 & 69 \\
\hline
\end{tabular}

${ }^{\mathrm{a}}$ Injections were made in CHIRALCEL OD-H column and the flow rate was $0.5 \mathrm{~mL} / \mathrm{min}$

${ }^{\mathrm{b}}$ Mobile phase used was 95:5 $n$-hexane/ isopropanol in all cases.

${ }^{\mathrm{c}}$ Reductions were carried out at $4{ }^{\circ} \mathrm{C}$. 